\title{
Philosophiques
}

\section{L'anthropologie des sciences : un programme pour la philosophie? (Entrevue avec Michel Serres)}

\section{Jean-Claude Simard}

Volume 14, numéro 1, printemps 1987

URI : https://id.erudit.org/iderudit/027005ar

DOI : https://doi.org/10.7202/027005ar

Aller au sommaire du numéro

Éditeur(s)

Société de philosophie du Québec

ISSN

0316-2923 (imprimé)

1492-1391 (numérique)

Découvrir la revue

Citer ce document

Simard, J.-C. (1987). L'anthropologie des sciences : un programme pour la philosophie? (Entrevue avec Michel Serres). Philosophiques, 14(1), 146-171. https://doi.org/10.7202/027005ar d'utilisation que vous pouvez consulter en ligne. 


\section{INTERVENTIONS}

Le 8 octobre dernier, le philosophe français Michel Serres était de passage au Québec pour y prononcer une conférence intitulée: "Culture humaniste et culture scientifique : existe-t-il une tierce voie? »

L'exposé avait lieu dans le cadre des conférences Augustin-Frigon, dont c'était la onzième, et se déroulait à l'École Polytechnique de Montréal. Organisé conjointement par l'École en collaboration avec la Fédération des Diplômés de Polytechnique, le tout était sous la responsabilité de Monsieur Dominique Piron, professeur de métallurgie, que nous voudrions remercier ici d'avoir permis et facilité l'entrevue dont le texte suit.

Après entente avec Monsieur Serres, il avait été convenu que la rencontre prendrait la forme du débat amical plutôt que de l'entrevue traditionnelle. Y ont participé : Jacques Daignault (U.Q.A.R., Sciences de l'Éducation) qui suit depuis plusieurs années déjà le travail de Michel Serres, Robert Nadeau (U.Q.A.M., philosophie) dont on connait l'intérêt soutenu pour l'épistémologie, Heinz Weinmann (Collège de Rosemont, philosophie), bien connu des lectrices et lecteurs du Devoir et qui publiera incessamment sur l'histoire du Québec un ouvrage qui doit beaucoup aux théories du philosophe français, et enfin, le responsable de la rubrique «Interventions » à titre de coordonnateur du projet.

Les quelques notes nécessaires à une meilleure intelligence du propos ont été reportées à la fin, de même que la bibliographie des nombreux ouvrages du philosophe, à laquelle on pourra se référer avec utilité.

J.-C. Simard. 


\title{
L'ANTHROPOLOGIE DES SCIENCES : UN PROGRAMME POUR LA PHILOSOPHIE ?
}

\author{
(Entrevue avec Michel Serres, 8-10-1986)
}

Philosophiques: Vous utilisez, dans Le Passage du NordOuest, une expression-choc qui a d'ailleurs été reprise dans la présentation de cet avant-midi. En guise d'introduction, $j$ 'aimerais que vous nous donniez des éclaircissements à ce propos. Il s'agit de cette expression où vous évoquez ceux qui s'affrontent dans notre société : d'une part, les «instruits incultes », et d'autre part, les «cultivés ignorants », Qu'est-ce à dire?

\section{LA DOUBLE SOLITUDE}

Michel Serres: Il s'agit plutôt d'un mot que d'un concept. Une expression, si vous voulez, où je plaisante un peu. Le Passage $d u$ Nord-Ouest a été écrit il y a relativement longtemps et l'expression est donc assez ancienne. J'avais été frappé il y a une vingtaine d'années par le fait que, comme nous en parlions d'ailleurs tout à l'heure après la conférence, une certaine tradition philosophique se développait tout à fait en dehors de la science et qu'au contraire, la tradition de l'enseignement scientifique se développait tout à fait en dehors de la philosophie. Cela créait un divorce. Or ce divorce n'est pas de tradition francophone. Celle-ci a toujours au contraire maintenu fermement l'alliance entre la science et la philosophie: tout le XVIII siècle français est encyclopédiste. Diderot était mécanicien tout autant qu'homme de lettres, Voltaire était newtonien et il traduisait les Principia tout en poursuivant la rédaction de ses divers écrits, et ainsi de suite. Plus tard, au XIX', Balzac sera l'ami intime de tous les savants de son temps, Auguste Comte élaborera une philosophie qui essaie 
d'incorporer les sciences en un système cohérent, Bergson et Valéry lui-même seront des penseurs complètement plongés dans la science de l'époque. En somme, il y avait là une sorte de mariage intime qui est notre tradition et ce mariage a été rompu par un divorce peu avant la Seconde Guerre mondiale. Dans l'enseignement, on a trouvé naturel que ceux qui faisaient des lettres ne sachent pas de science et que ceux qui faisaient de la science ne sachent pas du tout de littérature ou de philosophie. C'est en partie contre ce divorce que j'ai voulu m'insurger en créant l'expression dont vous faites état : d'un côté, les cultivés ne sont pas instruits de science et, d'un autre côté, ceux que l'on appelle savants ne sont pas cultivés.

Philosophiques: Et votre auvre consiste précisément à trouver des lieux de passage entre ces deux domaines...

Michel Serres: Des lieux de passage, oui. Exactement. Je ne fais aucunement ici ceuvre originale. Je tiens à rappeler, encore une fois, que je suis dans la tradition de langue française. On a peu idée à quel point, même avant le siècle dit des Lumières, chacun était déjà instruit de science. Molière, par exemple, ne savait-il pas par cour la physique épicurienne? La tradition rationaliste de langue française est une tradition d'alliance et cette alliance, je la reprends pour 1980, pour aujourd'hui. Mais je ne l'ai nullement initiée.

Philosophiques: De quand daterait approximativement le divorce?

Michel Serres: Il doit remonter, probablement, aux premiers écrits de Sartre. Vous savez comment il a commencé sa vie. Il disait: "Morale, peau de balle, science, trou de balle». À partir de lui et de sa vaste influence, le divorce a été établi et consommé.

Philosophiques: S'agit-il seulement d'une question d'individus ou ne se trouve-t-on pas aussi en présence d'une question d'institution?

Michel Serres: En fait, vous avez raison de le souligner, il s'agit surtout d'une question d'institution.

Philosopbiques: En ce sens, ne pourrait-on pas dire qu'à la limite, il faut remonter à Victor Cousin, c'est-à-dire à la façon même dont la philosophie s'est institutionnalisée et est entrée par la grande porte de l'Université? 
Michel Serres: Ce n'est sûrement pas faux. Mais, vous savez, quand j'ai passé l'agrégation de philosophie en 1952 , il était encore obligatoire d'avoir un certificat d'une licence de science au choix. Même alors subsistaient donc encore des traces de l'alliance en question.

Philosophiques: Et cela s'est complètement modifié depuis?

Michel Serres: Oui. Même institutionnellement, le divorce n'était pas alors complet. Mais il est vrai qu'il a été peu à peu accompli. Et puisqu'on parle d'institution, on peut poursuivre dans cette voie. On pourrait par exemple aisément évoquer des questions monétaires. Il y a d'un côté les Facultés et Universités qui coûtent cher, de l'autre, celles qui ne coûtent que très peu. $\mathrm{Au}$ Québec, on les appelle, je crois, «sèches ».

Philosophiques: Ou moins poliment, les «vaches à lait »...

Michel Serres: Voilà. On a peut-être mis de côté trop rapidement les disciplines peu dépensières.

Philosophiques: Pourtant, compte tenu du fait qu'on se trouve maintenant en présence d'un problème institutionnel, que cette ignorance mutuelle - ce qu'on pourrait appeler la double solitude - s'y est peu à peu sédimentée, il semble qu'un simple appel à la bonne volonté individuelle ne soit plus suffisant. À la limite, il faudrait examiner les programmes, la façon même dont les gens sont formés. En réalité, ne sommes-nous pas formés de manière ignorante?

Michel Serres: Tout à fait. Et puisque vous m'interrogez, je me permets de parler de mon expérience personnelle. J'ai eu la chance de faire d'abord des études de science - je suis licencié de mathématiques, j'ai fait, comme on dit en France, une "grande école scientifique " - et ensuite, j'ai abandonné pour des raisons, disons, d'éthique scientifique ou quelque chose d'approchant. Je suis tout de même de la génération qui a entendu Hiroshima et, comme chacun, je me suis longuement interrogé à ce moment-là. C'est d'ailleurs pour cela que j'ai commencé de faire de la philosophie. Donc, comme vous le voyez, ma vie entière m'a préparé à ce genre de choses, et je souhaite à beaucoup de gens de passer par ce type de tribulations, de les traverser. 


\section{HASARD ET VOYAGES}

Philosopbiques: Vous écrivez, Michel Serres, dans Le Passage du Nord-Ouest : "Il ne vaut pas la peine d'entrer jeune en philosophie si on n'a pas l'espoir, le projet ou le rêve de tenter un jour la synthèse. Le moins que l'on puisse essayer en ces lieux est le tour d'un monde ou les douze travaux d'Héraclès. Au moins cela quand on paye aussi cher en veilles, études et solitude. 》 Est-ce que ce projet encyclopédique, visant précisément à pallier la double solitude, n'a pas été depuis toujours à l'horizon de votre œuvre?

Michel Serres: Oui, c'est vrai. J'ai toujours été fasciné par les grands textes qui se présentaient comme des voyages, par L'Odyssée où Ulysse traverse de nombreux pays, fasciné aussi par les voyageurs de l'A mérique du Nord au moment de la découverte, par Jules Verne sur lequel j'ai fait un livre, etc. Vous voyez ce que je veux dire? Considérer que la philosophie est en quelque sorte une forme d'errance. Une errance de lieu en lieu, d'île en île, de mer en mer, et qu'il n'y a pas de philosophie sans cette sorte d'exploration des choses différentes.

Philosophiques: D'où l'utilisation de l'égide d'Hermès, le dieu-messager...

Michel Serres: Oui. Le dieu de la communication.

Philosophiques: Et sans doute aussi l'idée de randonnée qui chez vous est très importante : «at random »...

Michel Serres: Encore. Mais il faut ici préciser. Au MoyenÂge, le mot « randon » était un terme de chasse qui voulait dire le mouvement du cheval derrière un gibier qui essaie de le semer. Alors, le gibier que le chien et le cheval ensemble suivaient, faisait des mouvements à gauche, à droite pour échapper à ses poursuivants. Et on disait «courir à randon», ce qui signifiait courir très vite, un peu n'importe où, comme le gibier...

\section{Philosophiques: Au hasard...}

Micbel Serres: Au hasard. Et alors, à un certain moment, en traversant le Saint-Laurent ou la Manche, le mot s'est scindé. «Random» en anglais a voulu dire ces sauts hasardeux que faisait le gibier et « randonnée » a voulu dire chez nous le voyage. On s'est en quelque sorte partagés les deux sens. Et c'est un mot que j'aimerais reprendre en lui conservant sa double signification. 
Philosophiques: Comme la «noise», cet autre terme qui vous est cher et qui a subi une division similaire, comme vous l'avez montré dans Genèse?

Michel Serres: C'est aussi en effet un mot très curieux, dont est d'ailleurs issu la «nausée» si importante chez le Sartre des débuts...

\section{DE LA GRAMMAIRE ET DU STYLE}

Philosophiques: Dans le prolongement de la question initiale, celle des deux traditions, et relativement au projet encyclopédique et peut-être aussi à la manière de le réussir, vous avez tenu à préciser que l'expression de départ n'était pas un concept, mais un «mot ». À défaut de trouver une opposition entre mot et concept, n'y aurait-il pas là une différence qui témoigne des deux solitudes? Vous insistez constamment sur le retour aux mots pour en dégager notamment les concepts et je pense que votre œuvre témoigne manifestement d'un souci qui se rattache à la tradition française, un souci, disons, d'écriture. De ce point de vue, n'y aurait-il pas déjà, dans la manière dont vous écrivez, un mode se situant quelque part entre le mot et le concept, au sens où il y aurait déjà errance entre eux deux, comme une manière constitutive d'un style et peut-être même d'un genre?

Michel Serres: Vous me touchez beaucoup quand vous dites cela. J'ai longtemps cru et crois toujours que le renouveau d'une pensée passe nécessairement par la recherche d'une sorte de nouveau genre, de nouveau style. Et, à beaucoup d'égards, je ne suis pas d'accord avec un style philosophique inutilement technique. Il me semble par exemple que la recherche de la vérité, telle que la mène l'École analytique, avec un style extraordinairement pointilliste et découpé, est en soi une bonne chose, mais que la science, sur ce terrain, nous précède. Si l'on y songe bien, ce n'est peut-être pas tout à fait notre projet, à nous philosophes. Revenons à Platon qui demeure notre maître, quoi qu'on en ait. Chez lui, il y a, vous l'avez noté, deux hommes : le grammairien et le styliste. Le grammairien, c'est Socrate. Il veut parler court, il veut trancher, distinguer, opérer toujours des dichotomies.

Philosophiques: Ce serait l'homme de l'École analytique? 
Michel Serres: Voilà! Et, toujours, il arrête celui qui parle longuement. Mais il n'empêche que chez Platon, il y a quelqu'un qui, justement, parle longuement. Il y a celui qui écrit Le Banquet et les grands dialogues de la maturité. Ils y sont donc tous les deux. Le styliste et le grammairien : je crois qu'il n'y a pas de philosophie véritable sans ces deux hommes, sans Don Quichotte qui court après les moulins et Sancho Panza qui va tout doucement à dos d'âne.

Philosophiques: C'est une perspective fort stimulante. Alors, le passage qu'il faudrait trouver, en fin de compte, passage peut-être perdu, c'est le chemin de l'un vers l'autre?

Michel Serres: C'est cela. Entre le styliste et le grammairien... Vous savez, pendant de longs moments de ma vie, surtout dans ma jeunesse, $j$ 'ai beaucoup donné dans le texte très rigoureux, issu des mathématiques (j’avais beaucoup étudié Platon et l'histoire des sciences). Pourtant, à mesure que vient l'âge, en même temps qu'un certain type d'expérience, je verse tout doucement du côté du style. Je crois que cela aussi est une tradition francophone. Jamais nous n'avons eu en notre langue un philosophe qui ne soit en même temps un pur styliste. Montaigne est styliste, Pascal est styliste, oh combien!, Descartes même est styliste. La constante stylistique est véritablement au cœur de notre langue. Je m'explique. Cela va vous toucher comme Québécois. La langue anglaise m'a toujours paru semblable à une mer démontée tandis que le français m'a toujours fait l'effet d'un lac glacé. Il est en effet pauvre et précis alors que l'anglais est riche et flou. Par conséquent, l'Anglais a besoin de l'École analytique et nous de style. Pour des raisons opposées. Nous disposons déjà d'une langue analytique qu'eux ne possèdent pas. Par contre, une philosophie du style nous est nécessaire. Ces qualités respectives des deux langues sont en partie responsables de nos deux traditions philosophiques si divergentes. N'empêche que, depuis Platon, les deux types sont requis. Je crois profondément cela. D'un côté la rigueur, de l'autre, la recherche, en slalom entre le mot et le concept, que seul un styliste cisèle, que seule donne la pratique réelle de la langue.

Pbilosophiques: Est-ce qu'il n'y a pas là justement l'une des conditions réelles d'une intégrale encyclopédique qui dépasserait véritablement toute cueillette de données disparates? 
Michel Serres: Oui. Parce qu'en effet, les résultats passent souvent par la forme. J'en ai donné une illustration ce matin avec le thème de la religion: le problème des contenus nous divise et le problème de la forme nous associe.

Philosophiques: Est-ce la raison pour laquelle l'une des métaphores qui revient souvent dans votre œuvre, c'est le tissage, notamment le fil d'Ariane? Il s'agit, évidemment, d'allusions au célèbre tisserand...

Michel Serres: ... de Platon...

Philosophiques: ... et il s'agit aussi d'assembler, de tisser des choses qui, au départ, étaient séparées.

Michel Serres: C'est-à-dire que le tissage ne doit pas être une juxtaposition. Par exemple, ce matin, quand j'ai parlé de religion comme d'un système d'interconnexion entre des interactions ponctuelles, ce n'était pas un assemblage hétéroclite de deux problèmes qui se tirent la langue depuis le XVIII siècle. Une simple superposition entre science et humanités ne suffit pas. II faut bien sûr trouver l'opérateur qui permet le passage, l'entrelacs.

\section{OUVERTURE POUR UNE ANTHROPOLOGIE DES SCIENCES}

Pbilosophiques: J'aimerais revenir sur une question que vous avez évoquée à deux reprises déjà sous deux angles différents : le rapport entre philosophie et science d'une part et le rapport entre littérature et science, d'autre part. Vous faisiez allusion tout à l'heure au fait que les écrivains des XVII ${ }^{e}$, XVIII et $\mathrm{XIX}^{\mathrm{e}}$ siècles étaient très bien informés de la science de leur époque respective, en voulant comme preuve Diderot, Voltaire, Balzac, Molière même. Mais, pour s'en tenir pour le moment à la seconde dyade, n'existe-t-il pas aussi une tradition contraire à celle que vous évoquez et qui prétend s'enorgueillir d'une très franche opposition entre science et littérature? Je songe, par exemple, quittant les Écoles naturaliste ou positiviste à tout le Romantisme qui tient quand même le haut du pavé pendant tout le premier demi-siècle passé. Il est certain que Hugo, Vigny, Nerval ne sont pas d'abord préoccupés par leur rapport à la science, mais par l'exploration du rêve, par... 
Michel Serres: Il me faut vous ar rêter immédiatement. Vous savez que Vigny est l'auteur de poèmes philosophiques. Examinez-les de près et vous allez constater que s'y trouvent une analyse de la Révolution industrielle, de la machine à vapeur, de la locomotive : Vigny affiche clairement ses couleurs. Quant à Victor Hugo, je m'en tiendrai à un seul exemple très significatif. Il est un vers de lui que chacun connaît par cour : «Il élargit jusqu'aux étoiles le geste auguste du semeur». Avez-vous bien regardé ce vers? Est-ce que vous savez qu'il fut écrit l'année exacte où l'on découvrait la distribution aléatoire des étoiles? Étonnant, non?

Pbilosophiques: Pourrait-il s'agir d'une simple coincidence?

Michel Serres: Je ne puis savoir. J'affirme seulement que Hugo était suffisamment proche de ces questions pour avoir écrit au moment opportun quelque chose qui concernait manifestement l'astronomie de l'époque.

Philosophiques: Parviendrait-on aux mêmes conclusions si l'on examinait aussi l'autre dyade, le binaire science-philosophie? Il existe là aussi une longue tradition qui, prenant le contre-pied des Platon, Kant et Hegel, semble, à première vue, beaucoup plus soucieuse de se démarquer de la science que d'entretenir avec elle des relations d'amical cousinage. Elle peut s'autoriser entre autres de figures hautes en couleurs, telles Héraclite, Pascal, Nietzsche, pour ne nommer que ceux-là. On en retrouve d'ailleurs maintes traces chez un Heidegger que vous citiez ce matin encore.

Michel Serres: Il faut sûrement en excepter Pascal, qui est un inventeur forcené de géométrie, d'algèbre et de calcul infinitésimal...

Philosophiques: Exact. Mon oubli. Mais les autres, sont-ils irrémédiablement étrangers à l'approche que vous vous proposez?

Michel Serres: Malgré les apparences contraires, il faut bien convenir que non. Nietzsche avait pour amis les principaux thermodynamiciens de son temps et quand il crée la théorie du Retour éternel, il sait précisément de quoi il retourne à ce niveau.

Philosophiques: Et pourtant, ce n'est plus comme cela que l'on lit, goûte et interprète Nietzsche! 
Michel Serres: Voyez là sans conteste un effet pervers de notre divorce universitaire. Nous amputons Nietzsche, mais lui savait parfaitement la portée de ce qu'il écrivait.

Philosophiques : Et tout cela tient-il une place dans l'œuvre?

Michel Serres: Oui. Rappelez-vous les Lettres à Peter Gast. Elles montrent bien qu'il avait lu Clausius, Mayer, Thompson. D'ailleurs, puisqu'on évoque ces questions, il faut mentionner au passage que Freud avait lui aussi lu les thermodynamiciens, entre autres Maxwell. Mais revenons à la tradition dont vous faisiez état. Elle existe bel et bien et nous allons la reprendre de votre point de vue : elle m'intéresse beaucoup. Avez-vous remarqué que ce matin, j'ai cherché à exprimer quelque chose qui pourrait se résumer à peu près ainsi : nous savons ce qu'est l'histoire des sciences, nous connaissons l'origine du calcul des probabilités et pourtant, nous ignorons totalement ce que pourrait être une anthropologie des sciences? C'est précisément la discipline que je vous ai proposée pendant ma conférence. J'ai essayé d'en offrir deux ou trois illustrations. C'est une chose que le calcul des probabilités, c'en est une autre encore que l'histoire d'un tel calcul, mais quelqu'un a-t-il jamais eu la curiosité de jeter les yeux du côté d'une anthropologie religieuse du sacrifice? Là est, je crois, la racine originelle d'une telle connaissance. Voyons cela de plus près. Il arrive parfois que nous racontions de vieux récits. Par exemple, nous disons : «Ainsi, ils s'étaient assemblés autour de la femme adultère pour la lapider. Le Christ arriva et leur dit : "Que celui qui est sans péché lui jette la première pierre". » Nous lisons ce texte et pensons en nousmêmes: "Quelle magnifique leçon de morale!» Énorme méprise! Une morale? Pas du tout, mais pas du tout! Relisons attentivement et regardons ce que signifie «la première pierre». On s'aperçoit bien vite que le texte ne dit nullement ce que l'on croit habituellement.

Philosophiques: Que dirait-il alors?

Michel Serres: Si tu jettes la première pierre, c'est toi qui seras responsable de la première mort. Par conséquent, quelques années plus tard, tout le monde se retournera contre toi. Tu prendras alors la place de victime et on voudra à ton tour te lapider. Celui qui jettera la première pierre prendra plus tard ta place, et ainsi de suite. C'est le raisonnement du troisième homme. 
Peu à peu, toute la société s'acheminera vers sa destruction. Par conséquent, une loi stipulera: personne ne doit jeter la première pierre. C'est là quelque chose de très profond du point de vue de l'aveuglement sur la responsabilité, aveuglement que nous trouvons tous les jours dans les accidents de la route ou... les raisonnements scientifiques concernant le local et le global. Voilà donc un texte qui, pour nous, est de morale - traditionnellement de morale religieuse - et qui, lu selon le droit fil d'une autre optique, prend tout à coup une dimension extraordinaire: un aperçu sur une anthropologie de la connaissance.

Philosophiques: Parce qu'il manifeste une structure anthropologique cachée?

Michel Serres: Mais oui! Et il ne s'agit pas là d'un phénomène isolé. Ce qui semble d'abord tout à fait extérieur à la rationalité peut tout à coup se révéler comme la racine anthropologique très lointaine de nos connaissances scientifiques.

Philosophiques: Si ce texte, pour s'en tenir à celui que vous venez de citer, n'est pas reçu en fonction du message que vous y décodiez à l'instant, son efficace historique a-t-elle vraiment pu jouer en ce sens?

Pbilosophiques: Peut-être a-t-il été efficace dans l'exacte mesure où il n'était pas compris? Combien de choses dans la société sont efficaces à la condition qu'elles soient pratiquement inconscientes?

Philosophiques: Une espèce de «Ruse de l'Histoire »?

Michel Serres: Ou de la Raison...

Philosophiques: ...et qui serait cette «efficacité technologique » dont vous parliez ce matin...

Michel Serres: En effet. Pourquoi les sacrifices étaient-ils efficaces? Ceux qui s'y adonnaient ne savaient pas ce qu'ils faisaient, mais finalement, n'y avait-il pas chez eux des types d'efficacité qui, aujourd'hui, nous échappent, mais n'en représentent pas moins la racine anthropologique secrète de notre efficacité moderne?

Pbilosophiques: Est-ce qu'on pourrait trouver, envisageant l'histoire des sciences sous cet angle, une figure qui manifeste, 
d'une manière en quelque sorte éponymique, ce moment où l'on peut encore lire une anthropologie du sacrifice dans l'origine de la science moderne?

Michel Serres: Une figure éponymique... La chose serait sans doute assez difficile, mais on pourrait par contre déceler de nombreux exemples lacunaires. Parfois, je me demande si, dans $L e$ Passage $d u$ Nord-Ouest, dans cet effort que je fais pour réconcilier les deux solitudes, l'idée d'une anthropologie de la science n'est pas la seule chose qui, finalement, restera. Au fond, ce que nous appelons «humanités », ces mythes, contes et récits, ou encore ces romans, n'est-ce pas quelquefois le premier balbutiement de quelque chose de beaucoup plus profond? Se pourrait-il que les humanités soient notre anthropologie des sciences? La question me hante. Dans Les Cinq Sens, par exemple, j’ai de très longs développements sur la conception virginale du Verbe. Peut-être la chose vous a-t-elle frappé? Ce sont des dogmes appris dès l'enfance que nous en sommes venus à tourner en ridicule ; et puis, tout à coup, quand vous faites un tant soit peu de philosophie linguistique, vous êtes immédiatement frappés par la rigueur formelle de ce qu'on appelle un rite ou un mythe qui, soudainement... Mais, au fond, nous disons, plus bêtement, avec nos pseudorigueurs, ce qui a été énoncé là, de façon parfaitement paradigmatique!

\section{UNE PHÉNOMÉNOLOGIE DE LA SENSATION?}

Philosophiques: Puisque vous venez d'évoquer votre dernier ouvrage, Les Cinq Sens, pourriez-vous nous indiquer de quelle façon il se relie à vos actuelles préoccupations? A priori, il ne semble pas y avoir de rapport entre le projet encyclopédique dont nous nous sommes entretenus jusqu'à maintenant et le retour au fondement de la sensation, cette esthésie, si je puis dire, qui est à l'œuvre dans cet ouvrage. D'autant moins que vous avez déclaré était-ce une boutade? - qu'il s'agissait de votre "premier ouvrage de vraie philosophie».

Michel Serres: C'était en effet une boutade. Ce livre est écrit, pour la première fois peut-être chez moi, de manière presque polémique. Vous savez que pour l'École analytique et un certain type d'idéalisme linguistique, on ne peut percevoir que par le 
langage où la sensation est d'abord donnée. J'ai voulu protester quelque peu contre cette manière de voir. En effet, qu'on soit de l'École analytique, de l'École allemande ou de l'École française, depuis cinquante ans, on ne parle que de la parole, on écrit sur l'écriture, on discute du langage. C'est une erreur et $j$ 'ai voulu m'élever contre une telle procédure. La sensation est d'abord quelque chose de silencieux. C'était là mon premier motif polémique. Deuxièmement, j'ai voulu écrire sans appareil technique. Le moins possible, en tous les cas. Je crois que le mot transcendental est utilisé une seule fois, le mot analytique, deux. Je ne crois pas que s'y trouvent d'autres mots du même genre. De sorte que j'ai visé une langue qui soit complètement commune, qui permette tout. C'est là le lien que vous cherchez. Soit dit en passant, si vous lisez le dernier chapitre de l'ouvrage, vous verrez qu'il traite exclusivement de science, demandant : quel est le grand problème quand on sait suffisamment de science pour l'avoir complètement oublié ? Par conséquent, c'est bien toujours le même projet, mais il est, cette fois, presque à l'état blanc, un peu comme les physiciens affirment que toutes les couleurs se retrouvent dans la lumière blanche, cette «couleur » parfaitement transparente.

Philosophiques: Ne serait-ce pas l'espace quotidien qui surgit enfin comme tiers-inclus?

Michel Serres: Sans doute. Au fond, cette omni-liaison dont on parlait, je l'ai tout simplement retrouvée dans la vie de tous les jours.

Philosophiques: Serait-ce solliciter le texte indûment que d'y lire en sus l'appel de l'origine qu'on peut retrouver constamment présent dans toute votre œuvre? Quand, par exemple, dans le Lucrèce, vous évoquez cette primordiale chute liminaire des atomes ou quand, dans Genèse, vous parlez du « on » originaire... Ici, ne se trouve-t-on pas en présence d'une forme de phénoménologie originelle de la connaissance?

Michel Serres: Vous touchez là un point très profond. Il s'agit aussi en effet d'épistémologie, c'est-à-dire de partir à nouveau du fondement, comme dans trois ou quatre de mes autres ouvrages. 


\section{UNE ENTREPRISE DE RECHERCHE}

Philosophiques: Revenons, si vous le voulez bien, à votre conception d'une anthropologie de la science. La question qui me préoccupe est la suivante : si elle est efficace comme « technologie " dans la mesure exacte où elle n'est pas comprise, que se passera-t-il si on la transforme en programme de recherche? Ne risque-t-on pas alors de réduire cette «technologie » à une simple technique?

Michel Serres: Si. Mais vous savez... Je partage un peu làdessus l'opinion de Leibniz. Il disait: "Tout est intéressant, y compris les contes de bonne femme». Ou encore: «Il y a des veines d'or dans les rochers les plus stériles». Alors... Quant au danger, ne vous en faites pas, nous n'en sommes tout de même pas encore là !

Philosophiques: Danger est peut-être en effet un bien grand mot. La question sous-jacente, si je puis la reformuler, était en fait celle-ci : qui va effectuer un tel travail? Est-ce la communauté scientifique, retranchée dans sa solitude?

Michel Serres: C'est un travail qui nous incombe.

Philosophiques: «Nous»... Vous voulez dire les philosophes?

Michel Serres: Oui, c'est un authentique programme de philosophie. Et ce serait une admirable réconciliation entre les humanités et la philosophie rationaliste, elle qui, toujours, a eu soin d'établir cette espèce de rupture alors que, sans doute, l'Homme est enraciné dans ce dont elle tient tant à se séparer.

Philosophiques: Mais il reste que ce sera toujours un discours philosophique. Ne serait-ce pas alors une gageure vaine puisque les critères qui permettront l'appréciation d'une telle entreprise cognitive différeront toujours du fonctionnement et de la critériologie propres aux sciences? De sorte qu'une telle anthropologie reposerait sur un type d'exigence qui la leur rendrait difficilement accessible.

Michel Serres: Vous soulevez là en effet une sérieuse difficulté. Mais je prends la question dans un autre sens. Vous savez, on dit : «nous allons mourir».

Philosophiques: C'est ce que l'on sait. 
Michel Serres: Par conséquent toutes les entreprises de sauvetage sont bonnes. C'en est une. Vous voyez ce que je veux dire? J'étais à l'Université de New York il y a près de trois ans et, un jour, le président me reçoit à déjeuner. On venait alors de publier les dernières statistiques touchant la croissance des sciences et la décroissance des lettres. Sans en posséder le détail précis, c'est une chose que chacun connaît bien. Or, extrapolant la courbe, je me suis aperçu que nous étions voués à l'extinction aux alentours de 1992 ou 1993. Ce qui m'amena à dire au président : «ainsi, selon vos calculs, nous allons mourir en 1992 ou 1993 ? » Il m'a regardé un moment, a pris son verre et a répondu: «maybe before ». Peut-être avant... Et ça ne le gênait pas plus que cela. C'est la seule façon dont je puisse vous répondre.

\section{LA QUESTION DES SCIENCES HUMAINES}

Philosophiques: On en revient à la polarisation que vous déploriez au début de l'entrevue. Pour aborder une question qui n'a pas encore été effleurée, où situez-vous dans ce cadre ce qu'il est convenu d'appeler techniquement les «sciences humaines»?

Michel Serres: Elles sont passées avec le reste du côté des sciences. Vous avez visité l'École polytechnique où avait lieu ce matin la conférence, en particulier le laboratoire de pédagogie? C'est un laboratoire extrêmement important. Tous les professeurs s'y rendent puisqu'ils ont besoin de techniques d'enseignement. C'est un assez bon exemple de ce que j'affirme : ce qui s'est appelé autrefois sciences humaines et qui était aussi peu science que possible a traversé la barrière. Il ne reste plus de ce côté-ci, celui des lettres, que ce que nous appelions autrefois les «humanités ».

Philosopbiques: Et pourtant, quand on lit les textes philosophiques de l'heure, en particulier ceux traitant ces questions épistémologiques, on constate que bien peu sont enclins à reconnaître aux sciences humaines le même statut que celui qu'on veut bien reconnaître - puisqu'il s'affiche comme un fait - aux sciences de la Nature. On dit couramment encore maintenant que les sciences humaines sont en crise. On ne dit plus la même chose des sciences de la Nature. Elles ont indéniablement connu un problème de fondement qui s'est résorbé de l'intérieur sans se régler définitivement et avec lequel on a appris à vivre. 
Mais les sciences humaines, du point de vue même de ceux qui les subventionnent, ont à l'heure actuelle, un statut qui est hautement problématique.

Michel Serres: Mais comme vous le disiez tout à l'heure, ces problèmes sont de type institutionnel ou professionnel. Il n'y a plus de débouchés pour ces sciences, ou si peu.

Philosophiques: Il n'y en a pas davantage pour les philosophes, à vrai dire.

Michel Serres: Et cependant, je crois que c'est nous qui tenons le vrai filon. Je serais désespéré de devoir me limiter aux sciences. Pas vous? Les problèmes qu'ils traitent sont tellement locaux, tellement pointus, tellement parcellaires. Nous sommes encore les seuls fonctionnaires de la Totalité.

Philosophiques: Mais, n'est-ce pas là un paradoxe? On prétend - on l'affirmait déjà à l'époque où je poursuivais mes études, a fortiori aujourd'hui - qu'il n'est plus possible maintenant d'effectuer une telle totalisation du Savoir. Une telle Totalité, dit-on, n'est plus assignable.

- D'ailleurs, n'affirmez-vous pas dans une de vos œuvres, après Pascal, que «le grand Pan est mort»?

Michel Serres: Vous avez rencontré quelquefois des médecins, chers amis. Vous avez été soignés par des spécialistes du cœur, de la peau, ou autres. Vous ont-ils jamais guéri? Le bon vieux médecin de quartier, généraliste, l’a fait. C'est une espèce de plus en plus rare. (rires)

Pbilosophiques: Ce n'est pas lui en tout cas qui m'opérera!

Michel Serres: Non, mais c'est pourtant lui qui vous fera vivre. Je crois que le généraliste est toujours aussi essentiel. Le philosophe est ce médecin de quartier... ou devrait l'être.

Pbilosophiques: Mais, encore une fois, ce rêve est-il possible? Ou même seulement imaginable?

Michel Serres: Si l'on pose au départ une telle question, jamais on n'y arrivera! Vous vous rappelez celui qui disait qu'il n'était pas possible de marcher? Pourtant, il l'a fait. 
PHILOSOPHIE ET PÉDAGOGIE

Philosophiques: Est-ce que la philosophie n'est pas en train de devenir - pour moi cela n'a rien de péjoratif, bien au contraire! - une pédagogie? Ou pour le dire autrement, une médiation de pertinence?

Michel Serres: A-t-elle jamais cessé de l'être? Elle l'est maintenant. Elle l'était déjà chez les Grecs. Paideia, disaient-ils. À condition de conserver au mot pédagogie son sens éminemment profond, elle l'est bien certainement. En fait, ce que je vous propose en vous suggérant une relecture des textes anciens pour y déceler une anthropologie du Savoir, est-ce autre chose qu'une pédagogie? Le lien entre certains types de traditions. Qu'est-ce d'autre une pédagogie que ce transfert qu'un Heidegger appelait le "Traditional»? Ce statut accordé, j'abonde évidemment dans votre sens. La seule question au fond posée par Platon dans ses dialogues est: la vertu peut-elle s'enseigner? Paideia, toujours paideia.

Philosophiques: D'ailleurs, Socrate est-il autre chose que la pédagogie faite homme? Néanmoins, il faut reprendre la question. Vous parlez de "fonctionnaire de la Totalité». La formule est parente, bien sûr, de celle d'Auguste Comte: le philosophe comme «spécialiste des généralités». Un tel philosophe, dévoré, comme le dit magnifiquement Lévinas, par "l'inquiétude de la Totalité », ne devient-il pas - passons outre le paradoxe - un spécialiste comme les autres? Les scientifiques auront beau jeu de s'écrier: «les philosophes se spécialisant encore dans leurs généralités creuses. Laissons-les à leur studieuse oisiveté ! » Ne croyez-vous pas qu'ils passeront alors leur chemin?

SOCIOLOGIE DU POUVOIR :

SCIENCE ET/OU PHILOSOPHIE

Michel Serres: Vous parliez de crise des sciences humaines tout à l'heure. Je me demande plutôt s'il n'y a pas crise des sciences exactes, contrairement à ce que vous affirmiez. Je songe en particulier aux problèmes d'éthique, de déontologie des sciences médicales, par exemple dans le domaine de la reproduction humaine. N'entend-on pas plutôt les scientifiques s'écrier : «Mais 
nous ne savons plus ce que nous faisons! Au secours!» Et, se retournant, ils ne trouvent plus personne. C'est pourquoi ils s'adressent aux rabbins, aux curés, aux pasteurs, pour s'entendre répondre: "Aimez-vous les uns les autres ». Ça ne les intéresse pas beaucoup. Vers qui se tournent-ils alors? Mais vers les fonctionnaires de la Totalité justement ! Ils en ont besoin ${ }^{1}$. Ce fut très clair dans les années 79-80, au moment où les ministres euxmêmes décrochaient le téléphone en nous disant : "Où allonsnous trouver un nouveau droit de la reproduction?" Il fallait l'écrire. Si on rencontrait des problèmes au tribunal, qui appelaiton? Et la réponse était toujours: le philosophe. Vous voyez? Nous sommes véritablement indispensables à cette société nouvelle. Indispensables. Mais nous refusons de le voir.

Philosophiques: Il fut une époque où le philosophe surplombait le savoir, mais ce n'est plus possible aujourd'hui.

Michel Serres: Et pourtant c'est à nouveau ce qu'on exige de nous. Un jour, jai reçu un coup de téléphone du ministre de la Justice :

- Pouvez-vous passer me voir?

- Qu'y a-t-il?

- Il faut que j'écrive demain un droit pour les nouvelles sciences. Qui va m'aider?

Qui pouvait l'aider en effet? Quelqu'un qui soit capable de dire: «De la biologie? Soit. D'abord, la technique biologique. Ensuite les droits. Ensuite les religions. Ensuite les morales. Ensuite l'histoire. »

Philosophiques: Avez-vous l'impression que le philosophe n'a pas, sociologiquement, le crédit nécessaire pour agir ainsi? Je précise ma question. Beaucoup de gens - Foucault en particulier dans un article très documenté - s'interrogent sur la perte de pouvoir du philosophe...

Michel Serres: Tant mieux! (rires) Moins on a de pouvoir, mieux ça vaut! Plus on a de crédibilité.

1. Une bonne illustration de ce phénomène est fournie par le cas récent du savant français J. TeSTART. Voir à ce propos son dernier ouvrage L'CEuf transparent (Flammarion, «Champs », 1986) dont Michel Serres est justement le préfacier. 
Philosophiques: ... mais si cette perte de pouvoir prend la forme encore une fois d'une incapacité à effectuer la jonction des savoirs ? Est-ce que les scientifiques n'ont pas toujours prétendu à la philosophie, étendant presque de manière implicite leur domaine d'études? Je songe, par exemple, à Claude Bernard ou mieux encore à Darwin que Foucault, dans l'article auquel j'ai fait allusion, considérait comme une figure ouvrant en ce sens une ère nouvelle. Quand il développe des conséquences philosophiques de sa théorie...

Michel Serres: Je m'étonne que vous parliez ainsi de Darwin. Celui qui a développé les conséquences philosophiques de sa théorie, c'est Spencer. Darwin fait la science et Spencer fait l'idéologie (au sens positif du terme). Darwin n'est pas un philosophe à proprement parler.

Philosophiques: C'est exact. Mais Darwin écrit tout de même après L'Origine des Espèces, ouvrage éminemment scientifique, La Descendance de l'Homme, ouvrage à mi-chemin entre la philosophie et la science. Quant à Spencer, il est vrai qu'il propose une philosophie générale de l'évolutionnisme, mais il me semble que Darwin devait probablement classer ces prolongements dans la catégorie des "généralités creuses ».

Michel Serres: C'est en effet très possible. Mais revenons à votre question.

Pbilosopbiques: La nouvelle situation du savoir scientifique cristallisée chez Darwin ne se prolonge-t-elle pas encore? Par exemple, quand Monod écrit Le Hasard et la Nécessité, fait-il œuvre de science ou de philosophie? Quand Jacquard écrit Éloge de la Différence, se trouve-t-on en présence de théories scientifiques ou d'éthique? Est-ce que l'homme de science n'est pas, actuellement, et ce depuis Darwin, celui qui fait sa propre philosophie, son ontologie régionale en quelque sorte? Et le philosophe qui veut faire de même ne dispose-t-il pas de bien peu de crédit?

Michel Serres: C'est juste. Il est vrai que, sociologiquement parlant, à chaque période historique correspond celui à qui on s'adresse comme le titulaire du savoir important. On a vu, nous, pendant notre vie, cet homme-là changer de peau et de nature. Un 
temps, les gens se sont adressé à l'économiste comme au grand prêtre de la tribu. Ensuite le psychanalyste a rallié les suffrages. Maintenant ce serait plutôt le praticien des sciences positives. Pourtant, avez-vous remarqué comment leurs livres sont toujours curieusement décalés, frappés de caducité ? Beaucoup plus que les nôtres en fait. Parce que nous, quand nous nous piquons d'écrire sur les sciences, nous prenons en général la précaution d'étudier sérieusement le domaine dont nous traitons. Lorsque les savants font de la philosophie, ce sont eux qui se cantonnent en fait dans les "généralités creuses». Je pense, par exemple, à L'Homme neuronal de Jean-Pierre Changeux. C'est tout à fait pertinent en ce qui concerne la science actuelle, mais en ce qui a trait à la philosophie, ça doit refléter à peu près le début du XVIII siècle, peu avant La Mettrie. Le décalage est fabuleux. Évidemment, l'ouvrage a beaucoup de succès. Mais au point de vue du rapport entre science et philosophie, ça risque de durer ce que durent les roses. Pour ce qui est de Monod, je le situerais environ à l'époque de Camus. Vous savez, il m'a confié le manuscrit du Hasard et la Nécessité. C'est moi qui l'ai revu. Et quand je lui ai rapporté mes corrections, il m'a dit: "Tu n'as considéré que la biochimie. Je t'avais demandé de corriger la philosophie.» «Mais, lui ai-je répondu, libre à toi d'être camusien. C'est une chose qui te regarde, je n'ai pas à corriger ta philosophie. »

\section{L'INSTITUTIONNALISATION DE LA PHILOSOPHIE}

Philosophiques: Mais il y a tout de même une très nette crise de crédibilité du discours philosophique en Occident. Et si cette crise ne se résorbe pas très prochainement, on a l'impression que les philosophes auront de la difficulté à se faire entendre. Plus de publications, plus de subventions. À la limite, on assistera à une mort par attrition.

Michel Serres: Mais croyez-vous réellement que nous soyons un cas particulier? Socrate alla en tôle et fut exécuté. Et rappelez-vous encore l'âge classique! Descartes dut se réfugier en Hollande, Spinoza faillit se faire lapider, Voltaire s'est installé juste à la frontière...

Philosophiques: Vous avez raison. Mais il faut dire que la philosophie, à cette époque, n'était pas institutionnalisée comme 
elle l'est aujourd'hui. Les gens ont vécu cette institutionnalisation et on a l'impression que, de plus en plus, le regard qu'ils portent sur notre discipline, extrêmement critique, risque d'amener, finalement, cette situation un peu catastrophique qu'on n'ose imaginer. Déjà la difficulté de se faire entendre...

Michel Serres: Ce ne serait pas notre faute par hasard?

Pbilosophiques: Notre faute?

Michel Serres: Une philosophie qui consiste à décrire, pendant des années de cours, comme j'ai dû le subir, les quatre ou cinq éditions successives de La Recherche de la Vérité de Malebranche ou qui ressasse indéfiniment telle ou telle traduction d'A ristote...

Pbilosophiques: C'est un peu nos mathématiques...

Michel Serres: Il me semble que je n'ai jamais fait d'études de philosophie, si vous voulez que je vous dise la vérité. Vous comprenez? En philosophie, on ne m'a jamais enseigné la philosophie. On m'a toujours enseigné l'histoire de la philosophie, l'édition de la philosophie, l'édition de l'histoire de la philosophie, jusqu'à ce que je m'emmerde tellement que je ne veuille plus faire cela. Prolonger sa carrière en philosophie après une thèse sur Leibniz et les mathématiques ${ }^{2}$ en la répétant indéfiniment ? Non, merci. Oui, c'est vraiment notre faute!

Philosophiques: Est-ce que ça ne signifie pas au fond que ce que vous appelez la philosophie ne peut pas s'enseigner?

Micbel Serres: Si on enseigne indéfiniment la «Deuxième Méditation » de Descartes, il ne faut pas s'étonner des résultats, n'est-ce pas? L'institutionnalisation de la philosophie a souvent correspondu à sa mort.

Philosopbiques: Pour le Québec, en tout cas, la chose est claire. Lorsque vous parlez des éditions successives de $L a$ Recberche de la Vérité de Malebranche, je songe aux éditions successives de la Somme théologique de saint Thomas d'Aquin... Et cela a duré sous ce mode parodique pendant près d'un siècle!

2. Voir (ci-dessous) le premier ouvrage publié de Michel Serres (1968). 
Michel Serres: Vous savez, nous avons connu une scolastique équivalente en France. Il y avait le fonctionnaire Descartes, le fonctionnaire Nietzsche, le fonctionnaire Marx, etc. C'était l'objet d'études et on nous répétait indéfiniment Marx, Nietzsche, Freud, etc. jusqu'à plus soif. Mais ensuite, tout à coup, victoire! On a remplacé Marx par l'École analytique. Triste victoire. Au lieu de copier Marx, on les a copiés eux.

Philosophiques: Quel est alors l'impact d'une telle situation sur le mode d'institutionnalisation de la philosophie ? Qu'en est-il des programmes, de l'agrégation, ainsi de suite?

Michel Serres: Je l'ignore. Vous savez peut-être que je n'enseigne plus depuis dix ans. Que dis-je ? Depuis quinze ans! On m'a foutu dehors.

Philosophiques: Mais vous êtes sûrement très bien informé. Pour vous, une telle situation n'est certainement pas très prometteuse?

Michel Serres: Bien sûr que non! Et elle s'aggrave constamment. Je crois que la philosophie se vit ailleurs.

Philosophiques: Vous voulez dire en dehors de l'institution?

Michel Serres: De toute façon, la philosophie a toujours été marginale. La différence, c'est qu'en ce moment, elle s'automarginalise. Le père Nietzsche a été un marginal de l'Université allemande toute sa vie. Bergson était à peine supporté en France. À l'époque, le Collège de France était une voie de garage. Je crois que l'histoire de la philosophie a quelque chose à voir avec l'histoire des religions. Comme le savait Bossuet, celle-ci ne fait des progrès que par ses hérésies ${ }^{3}$.

\section{PHILOSOPHER EN FRANÇAIS}

Philosophiques: Est-ce que votre projet d'éditer le Corpus des philosophes français est une façon de sortir la philosophie de sa marginalité?

3. Plaisante allusion à L'Histoire des variations des Églises protestantes de Bossuet qui, il va sans dire, ne l'entendait pas tout à fait ainsi... 
Michel Serres: C'est une autre question. Vous savez que le tout se déroule très bien. Près de 39 volumes sont maintenant parus dont, parmi les derniers, le Descartes et le Bodin en six volumes. On a aussi édité Arnauld, D'Alembert, etc. On ne fait pas fortune mais ça marche bien. Pour ce qui est de l'origine du projet, puisque vous voulez la connaître, elle est ailleurs. Et en tant que Québécois, elle va, je crois bien, vous intéresser. Savez-vous que, depuis que je suis entré en philosophie, je n’ai jamais assisté en France, à une thèse sur la philosophie de langue française? Chez nous, il y a ceux qui s'occupent de la tradition allemande, ceux qui s'occupent de la tradition anglo-saxonne, et il y a les sciences humaines. Celles-ci sont en général dominées soit par la sociologie américaine, soit par la psychanalyse de Freud. Par conséquent, on assistait à un enterrement presque total de la philosophie de langue française depuis environ les années 35. C'était chose morte. Il était même tout à fait normal de dire que qui parlait la langue française n'était pas philosophe. Ayant vécu cela, j'ai juré, à l'approche de la trentaine que, vieux, j'essayerais de modifier ce cap. Et c'est là l'origine de mon Corpus. C'est, si vous me prêtez l'expression, une révolte péquiste. Plus de philosophie de langue française... Ce qui arrivait alors à la philosophie me rappelait ce qui était aussi arrivé à la musique française au début du XIX ${ }^{\mathrm{e}}$ siècle : sous l'influence de Rousseau, on l'avait rejetée complètement au profit exclusif de la musique italienne. Il en allait de même pour la philosophie et j'ai voulu la ressusciter. C'était pour moi comme un devoir sacré envers la langue. Vous savez qu'un professeur de la Sorbone dont je tairai ici le nom - je n'ai pas l'habitude de nommer les gens dont je dis du mal - a écrit dans une revue célèbre en France un article intitulé : «Why I am not a French philosopher ».

\section{Pbilosopbiques: Est-ce « off the record»? (rires)}

Michel Serres: Je voudrais surtout que ça n'ait jamais été écrit. Je ne suis nullement xénophobe, mais on pourrait de temps en temps philosopher dans notre langue.

Philosophiques: Vous avez évoqué à l'instant le fait que nous soyons Québécois. D'autre part, vous faites allusion à la tradition philosophique nationale... 
Michel Serres: Non, pas «nationale». Linguistique. Parce que la langue française, ce n'est pas la langue de la nation appelée France. C'est une langue qui... Enfin, ce n'est tout de même pas à vous que je vais expliquer ça! (rires)

Philosophiques: Ma question portait, elle, sur une tradition linguistique et nationale, justement. Il me semble que la situation de la philosophie québécoise, telle qu'on peut la percevoir à la suite d'un survol rapide, est assez similaire à la situation du savoir décrite dans vos ouvrages. Vous parlez de la nécessité d'établir une tierce voie, un tiers-inclus entre les humanités et les sciences exactes. Or, il se trouve que nous sommes continuellement confrontés, nous du Québec, à une culture française plus humaniste d'une part, et, d'autre part, à une influence américaine qui est, elle, plus scientifique. En conséquence, plusieurs croient que, si la philosophie québécoise a une originalité, c'est celle de chercher une tierce voie. On la perçoit souvent comme une tentative pour trouver un moyen terme entre deux traditions opposées. Par ailleurs, l’ouvrage où vous abordez spécifiquement la question d'une tierce voie est dédié «aux amis du Québec » et situé au Canada, c'est-à-dire en un lieu géographique appelé «le passage du Nord-Ouest ». S'agit-il là de simples coïncidences?

Michel Serres: Vous savez, pour des raisons circonstantielles, j'ai une affinité certaine pour la situation québécoise. D'abord - et la chose risque de vous surprendre - je suis aussi loin de la tradition de langue française que vous. Je suis né dans le Sud-Ouest et, à la table familiale, on parlait la langue d'Oc et non pas le français. En somme, une langue de souche française mais dérivée, un peu comme la vôtre. De sorte que cette situation de marginalité par rapport au centre de la langue française, je l'ai connue tout comme vous. Par conséquent, pour moi, c'est là quelque chose d'assez profond ${ }^{4}$. Mais ce pourquoi j'ai surtout

4. “Pour moi le Québec, c'est plusieurs fois l'enfance. Mon enfance. D'abord, parce qu'étant très jeune, j'avais lu beaucoup de littérature sur cetre question et qu'un de mes désirs les plus secrets lorsque j'étais enfant, c'était de connaître ce pays, de voyager dans ce pays, de connaître les gens du pays et leur histoire surtout qui, d'une certaine manière, nous avait échappé. J'avais la sensation que dans ce pays-là il y avait un secret que la France avait perdu. Et lorsque j'y suis allé, je n'ai pas été déçu du tout. (...) Je suis fils de marinier, fils de paysan marinier. (...) Ce rapport au monde, ce rapport au fleuve, ce rapport à la nature, ce rapport au travail, je le retrouvais intact (au Québec). 
appelé cet ouvrage "Passage du Nord-Ouest», c'est que, lisant Chateaubriand... Vous savez qu'il était parti ici faire de l'ethnologie? Et cet espèce d'écheveau extraordinairement complexe dans l'espace et dans le temps m'avait séduit: je crois que le problème d'une tierce voie est métaphoriquement copié sur cette curieuse géographie du Grand Nord canadien. Il faut dire aussi que j'ai écrit une partie de cette œuvre ici, à l'époque où j'étais visiteur à l'Université de Montréal. Comme vous le voyez, c'était donc plus qu'une coincidence et moins qu'une nécessité.

Pbilosophiques: Je crois que les lectrices et lecteurs d'ici auront bien perçu cet entre-deux qui reflète une fois de plus une caractéristique centrale de votre ouvre. Sans doute y verront-ils une bonne façon de naviguer une dernière fois dans les eaux peu fréquentées «du Nord-Ouest». Michel Serres, merci pour cette agréable «randonnée » sous l'égide d'Hermès.

(...) J'ai beaucoup plus appris dans des endroits comme l'Île aux Coudres que dans n'importe lequel livre, n'importe laquelle bibliothèque. »

Témoignage de Michel Serres tiré du film que Jean-Daniel Lafond consacrait tout récemment à l'ouvre de Perrault: Les traces du rêve. Monsieur Serres y intervient à plusieurs reprises à titre d'ami du cinéaste Perrault. La première du film avait lieu à Montréal dans la soirée du 8 octobre, journée même où fut prononcée la conférence à l'École Polytechnique et réalisée l'actuelle entrevue. 


\section{OUVRAGES DE MICHEL SERRES}

\section{Aux Presses universitaires:}

Le Système de Leibniz et ses modèles mathématiques, 2 vol., 1968. Réédition en un volume, 1982.

Aux éditions de Minuit :

Hermès I. La communication, 1969.

Hermès II. L'interférence, 1972.

Hermès III. La traduction, 1974.

Hermès IV. La distribution, 1977.

Hermès V. Le passage du nord-ouest, 1980.

Jouvences. Sur Jules Verne, 1974.

La Naissance de la physique dans le texte de Lucrèce. Fleuves et turbulences, 1977.

\section{Aux éditions Grasset :}

Feux et signaux de brume. Zola, 1975.

Le Parasite, 1980.

Genèse, 1982.

Rome. Le livre des fondations, 1983.

Les cinq sens. Philosophie des corps mêlés-I, 1985.

Aux éditions Hermann :

Esthétiques. Sur Carpaccio, 1975

Auguste Comte. Leçons de philosophie positive, tome I, 1975.

\section{Aux éditions Flammarion :}

Détachement, 1983. 


\section{PRÉSENTATION}

La dernière livraison de Pbilosophiques contenait deux conférences prononcées lors des États Généraux de la philosophie tenus en 1984. En effet, comme nous l'expliquions alors, le projet de réunir en un seul volume l'ensemble des exposés présentés lors de cet important événement avait malheureusement dû être abandonné par les responsables. Nous annoncions à ce moment qu'une troisième conférence, "La responsabilité de la clarté, » figurerait au sommaire de l'actuel numéro. On la trouvera ci-après. Pour l'instant, il s'agit du dernier texte issu de cette source et nous ne prévoyons pas en publier d'autres dans un avenir rapproché.

Comme l'avaient fait aussi les deux auteurs précédents, Claude Panaccio a préféré conserver à son intervention son style originel, fonction d'une transmission verbale. Souhaitons cette fois encore que lectrices et lecteurs sauront y retrouver un peu de cette effervescence qui avait profondément marqué la tenue des États Généraux de la philosophie au Québec.

J.-C. Simard 